\title{
Preclinical safety assessment of glycosides based standardized fenugreek seeds extract: Acute, subchronic toxicity and mutagenicity studies
}

\author{
Pallavi Deshpande, Vishwaraman Mohan, Prasad Thakurdesai* \\ Indus Biotech Private Limited, Kondhwa, Pune, Maharashtra, 411048, India.
}

\begin{tabular}{|c|c|}
\hline ARTICLE INFO & ABSTRACT \\
\hline Article history: & \multirow{10}{*}{$\begin{array}{l}\text { Objective: To evaluate acute oral toxicity (AOT), subchronic toxicity, and mutagenic potential of glycosides } \\
\text { based standardized fenugreek (Trigonella foenum graecum L.) seeds extract (SFSE-G). Materials and Methods: } \\
\text { The AOT, subchronic ( } 90 \text {-day repeated dose) toxicity and mutagenicity (reverse mutation test) of oral } \\
\text { administration of SFSE-G were evaluated using Sprague-Dawley (SD) rats as per OECD guideline no. } 423 \text {, No. } \\
408 \text { and } 471 \text { respectively. Results: The SFSE-G did not show mortality or treatment-related adverse signs during } \\
\text { acute (limit dose of } 2000 \mathrm{mg} / \mathrm{kg} \text { ) and subchronic }(90-\text { days repeated dose of } 250,500 \text { and } 1000 \mathrm{mg} / \mathrm{kgwith} 28 \\
\text { days of recovery period) administration. The SFSE-G showed oral median lethal dose }\left(\mathrm{LD}_{50}\right) \mathrm{more} \text { than } 2000 \\
\mathrm{mg} / \mathrm{kg} \text { during AOT study. The no-observed adverse effect level (NOAEL) of SFSE-G was } 1000 \mathrm{mg} / \mathrm{kg} \text { in male } \\
\text { rats and } 500 \mathrm{mg} / \mathrm{kg} \text { in female rats during subchronic toxicity study. Furthermore, SFSE-G did not show } \\
\text { mutagenic potential in vitro. Conclusions: SFSE-G was found safe for acute and subchronic ( } 90 \text { days repeated } \\
\text { dose) administration in rats with no mutagenic potential. }\end{array}$} \\
\hline $9 / 03 / 2016$ & \\
\hline $07 / 2016$ & \\
\hline $\mathrm{d}$ on: $08 / 08 / 2016$ & \\
\hline Available online: $26 / 09 / 2016$ & \\
\hline Key words: & \\
\hline & \\
\hline acute oral toxicity; & \\
\hline & \\
\hline & \\
\hline
\end{tabular}

\section{INTRODUCTION}

There is increasing awareness and general acceptability of the use of herbal drugs in today's medical practice (Upton et al., 2016) Most of the natural products or herbal medicines contain a diverse variety of phytoconstituents because of variation in growth pattern, geographical location, time of harvesting and storage (Yau et al., 2015). Correct identification of composition of herbal medicine is essential to ensure consistent quality, safety and efficacy (Upton et al., 2016). The process of standardization is used to prescribe a set of standards or inherent characteristics with definitive qualitative and quantitative values thatare indicative of consistent quality and reproducibility. Apart from consistent quality, all medicines need to fulfill the basic requirements of safety. However, the safety of herbal medicines has become a major concern to both regulatory

\footnotetext{
* Corresponding Author

Prasad Thakurdesai, Indus Biotech Private Limited, Kondhwa, Pune, Maharashtra, 411048, India. Email: prasad @ indusbiotech.com
}

health authorities and the general public (Kalaiselvan et al., 2015, Starr, 2015). Therefore, safety or toxicological studies on herbal medicines needs to be conducted using well accepted international guidelines to ensure safe use for long-term human consumption. One of the potential natural sources of nutritional and medicinal applications is fenugreek (Trigonella foenum-graecum L.) seed. Fenugreek seeds are being used by people in Asia, Africa and Mediterranean countries as one of the ingredients in daily diets (Ulbricht et al., 2008).

Past reports on fenugreek seeds highlighted a variety of medicinally active phytoconstituents namely alkaloids and polysaccharides (Petropoulos, 2003), flavonoids (Huang and Liang, 1999, Wagner et al., 1973), triterpenoids (Shang et al., 1998) and steroidal sapogenins (Sauvaire et al., 1991).Safety of fenugreek seeds in powder form in human has been established in many clinical trials and reviews (Basch et al., 2003). Fenugreek seeds are certified as GRAS (Generally recognized as safe) item under clause $\$ 182.20$ (Essential oils, oleoresins and natural extractives including distillates) by US Food and Drug Administration (US FDA). 
One of the important phytoconstituent of fenugreek seeds is glycosides content. These include a variety of furostanol (Gupta et al., 1985, Kang et al., 2013, Murakami et al., 2000, Yoshikawa et al., 1997) and flavonol (Han et al., 2001, Pang et al., 2012b, Rayyan et al., 2010, Taylor et al., 2000) glycosides. Fenugreek seeds glycosides are known to have androgenic and anabolic (Aswar et al., 2010), anti-inflammatory and anti-melanogenic (Kawabata et al., 2011), platelet aggregation inhibition (Pang et al., 2012a) and anti-oxidant (Kenny et al., 2013) properties. The androgenic and anabolic activity (Aswar et al., 2014) and subacute toxicity (Kandhare et al., 2015) of glycosides based standardized fenugreek seeds extract (SFSE-G) in male rats has been reported . Androgenic benefits of SFSE-G capsule supplementation is reported in sedentary (Mokashi et al., 2014) and college-age men (Wilborn et al., 2010). However, the evidence of long-term safety for SFSE-G is not being available.

In order to explore clinical potential of SFSE-G for longterm human consumption, toxicology testing in laboratory animals using well-accepted international guidelines is required. Therefore, the present study aimed to evaluate acute oral toxicity (AOT), repeated dose (90-days, subchronic) toxicity in laboratory rats and mutagenicity testing using OECD guidelines

\section{MATERIALS AND METHODS}

\section{Animals}

Male and female Sprague-Dawley (SD) rats were used for the acute and subchronic toxicology studies. The rats were obtained from the animal house of Indian Institute of Toxicology, Pune, India. The animals were acclimatized to laboratory conditions for 7 days prior to the experiments. The rats were maintained at a room temperature of $22-24^{\circ} \mathrm{C}$, relative humidity between $30 \%$ and $70 \%$; $10-15$ air changes per hour with a $12 \mathrm{~h}$ light/dark cycle.

During acclimatization, the animals were housed in polypropylene cages provided with a standard pellet diet $(\mathrm{M} / \mathrm{s}$. Nav Maharashtra Chakan Oil Mills Ltd., Pune) and water ad libitum. All protocol was approved by Institutional Animal Ethics Committee of Indian Institute of Toxicology, Pune, India.

All the animal experimentations were performed as per Committee for the Purpose of Control and Supervision of Experiments on Animals (CPCSEA) guidelines in accordance with "Principles of Laboratory Animal Care" (NIH publication no. 8523, revised 1985).

\section{The test compound, SFSE-G}

The test compound, SFSE-G, is a standardized extract of fenugreek seeds (containing $89.42 \%$ of glycosides), prepared and standardized by HPLC using earlier reported procedure (Kandhare et al., 2015)and provided for the study by Indus Biotech Private Limited (Pune, India). The solution of SFSE-Gwas freshly prepared in distilled water to obtain dose volume of $10 \mathrm{ml} / \mathrm{kg}$ body weight of rats. SFSE-G solution in sterile water for injection was used for mutagenicity evaluation.

\section{Acute oral toxicity (AOT) study}

The acute toxicity study of SFSE-G was performed according to OECD guideline 423 (Organisation for Economic Cooperation and Development, (Organisation for Economic Cooperation and Development, 2002). The rats were grouped in 2 groups (5 rats/sex/group) and treated with a single dose as follows:G1 - Vehicle control (VC) (double distilled water, 10 $\mathrm{mL} / \mathrm{kg}$, oral), and G2 (SFSE-G, $2000 \mathrm{mg} / \mathrm{kg}$, oral.). The rats were observed daily for 14 days following administration for mortality and clinical signs of toxicity. On day 15 , all rats were euthanized and underwent gross pathological examination for signs of toxicity via necropsy.

\section{Subchronic (90-day repeated dose) toxicity study}

The study complies with the OECD Guideline for the testing of Chemicals No.408 (Organisation for Economic Cooperation and Development., 1998).The separate vehicle control (VC) group was maintained on distilled water as designated as G1. Based on median lethal dose $\left(\mathrm{LD}_{50}\right)(2000 \mathrm{mg} / \mathrm{kg})$ obtained from AOT study, animals were orally administered daily once with following treatments:

G1 - VC (Distilled water, $10 \mathrm{~mL} / \mathrm{kg}, 90$ days) - 15 animals per sex

G2 - SFSE-G-250 (SFSE-G, $250 \mathrm{mg} / \mathrm{kg}$, 90 days) - 15 animals per sex

G3 - SFSE-G-500 (SFSE-G, 500 mg/kg, 90 days) - 15 animals per sex

G4 - SFSE-G -1000 (SFSE-G, 1000 mg/kg, 90 days) - 15 animals per sex

G1R - VC reversal group -VC-R - (Distilled water, 10 $\mathrm{mL} / \mathrm{kg}, 119$ days) - 10 animals per sex

G4R - SFSE-G -1000-Reversal group (SFSE-G-1000-R) (SFSE-G, $1000 \mathrm{mg} / \mathrm{kg}$ for 90 days) followed by (distilled water, $10 \mathrm{~mL} / \mathrm{kg}$ (from day 91 to day 119) - 10 animals per sex

All treated rats were observed daily for mortality and clinical signs during the 90-day study period whereas reversal groups (G1R and G4R) were observed for upto day 119 (Reversal period of 28 days). The eyes of control and all the treated dose group animals were examined prior to the initiation of the dosing and on day 91 and 119 (for G1R and G4R) of the study. Eye examination was carried out using an ophthalmoscope (mini 2000, HEINE Optotechnik, Herrsching, Germany) after induction of mydriasis with $0.5 \%$ solution of tropicamide. The body weight and feed consumption of each rat were recorded at weekly intervals throughout the study period. Towards the end of the exposure period (day 91), functional observations (by grading different sensory reactivity to stimuli of different types (auditory, visual and proprioceptive stimuli), assessment of grip strength (digital grip 
strength meter (Columbus Instruments International, Columbus, $\mathrm{OH}$, USA) and motor activity measurement were performed. The animals were placed in metabolic cages overnight and urine excreted by each animal was collected on day 91 and on day 119 (G1R and G4R) of the study for urinalysis. The parameters for urinalysis included: specific gravity, $\mathrm{pH}$, occult blood, protein, bilirubin, ketones, glucose, nitrite, and urobilinogen. Using whole blood, hematological and coagulation analyzes were carried out. The parameters for hematological analysis included: red blood cell count (RBC), reticulocyte count, hemoglobin $(\mathrm{Hb})$, hematocrit (HCT), mean corpuscular hemoglobin (MCH), mean corpuscular hemoglobin concentration (MCHC), mean corpuscular volume (MCV) and total leukocyte count (TLC), \% cells in differential leukocyte count (DLC) (including lymphocyte (L), monocyte (M), basophils (B), eosinophils (E), and Prothrombin time (PT). Additionally, clinical chemistry was evaluated including Blood Urea Nitrogen (BUN), Alanine Aminotransferase (ALT), Aspartate Aminotransferase (AST), Alkaline Phosphatase (ALP), Gamma Glutamyl Transferase (GGT), Creatine Phosphokinase (CK), Lactate Dehydrogenase (LDH), Fasting plasma glucose (FPG), Calcium (Ca), Phosphorus (P), Bilirubin, Albumin, creatinine (CR), Sodium (Na), Potassium $(\mathrm{K})$, Chlorine $(\mathrm{Cl})$, Cholesterol, and Triglycerides (Trig). On day 91, all animals were euthanized and underwent gross pathological examination for signs of toxicity via necropsy.

All organs, mucosa, body cavities, etc. were examined for gross pathological changes. Major organs and major endocrine glands (pituitary, adrenal, thymus, thyroid, sex, etc.) were weighed and absolute and relative weights (i.e. percent of the body weight) were calculated. Tissue samples from selected organs (heart, kidney, liver, lung, spleen, stomach, pancreas and skeletal muscle) from the VC (G1 and G1R) and $1000 \mathrm{mg} / \mathrm{kg}$ (G4 and G4R) were preserved, fixed, and stained for histopathological evaluation via light microscopy. The remaining tissues/organs were preserved in $10 \%$ neutral buffered formalin from control and different dose groups.

\section{Mutagenicity study}

Mutagenicity was evaluated by bacterial reverse mutation test (AMES test) was performed in full compliance with the OECD guidelines for mutagenicity testing namely Test No: 471 (Organisation for Economic Co-operation and Development, 1997). As no significant cytotoxic effect was observed, the five highest doses were then used in the subsequent mutagenicity evaluation.

To evaluate mutagenicity, five strains of histidinedependent Salmonella typhimurium (TA97a, TA98, TA100, TA1535 and TA102) were tested in triplicate at the five highest doses $(5000.00,1666.67,555.55,185.18$ and $61.72 \mu \mathrm{g} /$ plate) of SFSE-G. Rat liver homogenate tested with mutagen 2aminofluorene before use. Metabolic activation was performed using a cofactor-supplemented post-mitochondrial fraction (S9 fraction). Positive controls (2-aminofluorene, 2-aminoanthracene, methyl methanesulfonate, 4-Nitroquinolene-N-Oxide, danthron and sodium azide) with and without S9 activator and negative controls (VC and phosphate buffer) with and without S9 activator were included in the evaluation. This was done to ensure the test system was functioning properly (positive controls) and to obtain baseline revertant frequencies for the various strains of bacteria used in the study (negative controls). The plates were counted after $48 \mathrm{~h}$ of incubation at $37{ }^{\circ} \mathrm{C}$. The mutagenic activity of the test substance was considered for positive in case of increased concentration over the range tested and a reproducible increase at one or more concentrations in a number of revertant colonies per plate in at least one strain with or without metabolic activation system.

The test substance was considered to be toxic if there was a decrease in the number of revertants and/or thinning or absence of background lawn.

\section{Statistical analysis}

Statistical analysis was performed using SPSS analysis program for windows Version 17.0 (SPSS Inc, USA). All the data were checked for normality and values were represented as mean \pm standard deviation (SD). Data of body weight and food intake was analyzed by two-way ANOVA followed by unpaired ' $t$ ' test. Remaining parameters were analyzed by separate One way ANOVAs.

Dunnett's test was used to analyze the differences between treated groups with respective $\mathrm{VC}$ groups (G2, G3, G4 v/s. G1 and G4R v/s.G1R). The value of $\mathrm{P}<0.05$ was considered to be statistically significant.

\section{RESULTS}

\section{Acute oral toxicity (AOT)}

None of the rats died during the observation period. No clinical signs of toxicity, such as ill health or abnormal behavior, occurred during the study. There were no abnormal observations in body weight of rats. No abnormalities or pathological changes were observed in the necropsy. Under the experimental conditions, the results indicated that the median lethal dose $\left(\mathrm{LD}_{50}\right)$ of SFSE-G is greater than $2000 \mathrm{mg} / \mathrm{kg}$ of body weight.

\section{Subchronic (90-day repeated dose) toxicity}

Daily oral administration of SFSE-G for 90 days did not induce any obvious symptom of toxicity in rats of any sexes, up to the highest tested dose $(1000 \mathrm{mg} / \mathrm{kg})$. No deaths or obvious clinical signs were found in any groups throughout the study period. Body weights of male and female rats did not show significant difference during treatment period except for SFSE-G1000 group in female rats on 90 days period and SFSE-G-1000-R group (v/s. G1R group) (Table 1). No gross abnormalities were observed in necropsies of any of the rats. The food and water consumptions were measured throughout the study and did not significantly change as compared to the VC rats (Figure 1). Ophthalmoscopic examination and functional observation tests revealed no abnormalities attributable to the treatment. 

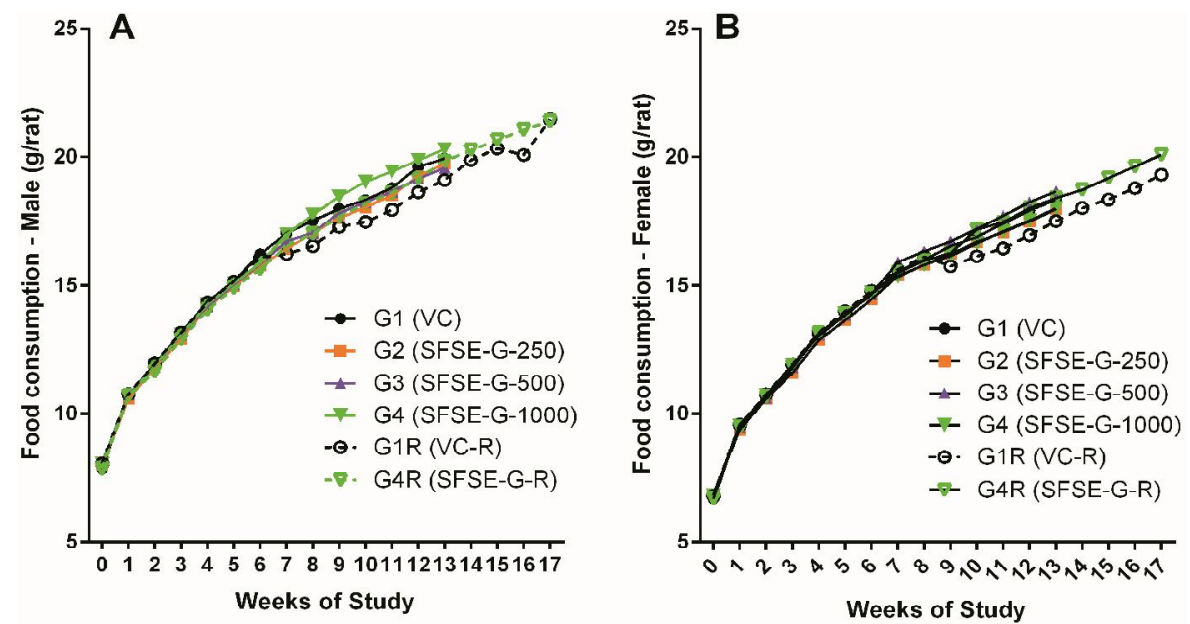

Fig. 1: Effect of SFSE-G food consumption in (A) Male and (B) Female rats during 90 days repeated dose toxicity study. Data are expressed as mean \pm standard deviation $(\mathrm{SD})$.

Table 1: Effect of SFSE-G on body weights of rats during 90 days repeated dose toxicity study.

\begin{tabular}{|c|c|c|c|c|c|c|}
\hline \multirow{2}{*}{ Weeks } & \multicolumn{6}{|c|}{ Group } \\
\hline & G1 & G2 & G3 & G4 & G1R & G4R \\
\hline & VC & SFSE-G-250 & SFSE-G-500 & SFSE-G-1000 & VC-R & SFSE-G-1000-R \\
\hline \multicolumn{7}{|l|}{ Male } \\
\hline 0 & $85.67 \pm 7.17$ & $83.06 \pm 3.41$ & $83.55 \pm 3.82$ & $84.47 \pm 5.08$ & $83.94 \pm 6.20$ & $85.26 \pm 6.00$ \\
\hline 12 & $374.11 \pm 29.51$ & $376.29 \pm 38.54$ & $372.69 \pm 38.28$ & $352.49 \pm 37.58$ & $364.78 \pm 30.37$ & $363.53 \pm 31.67$ \\
\hline 13 & $381.14 \pm 28.45$ & $382.15 \pm 37.47$ & $377.23 \pm 35.91$ & $358.42 \pm 37.42$ & $371.31 \pm 29.78$ & $369.30 \pm 30.04$ \\
\hline 17 & & & & & $391.72 \pm 25.46$ & $374.86 \pm 29.63$ \\
\hline \multicolumn{7}{|l|}{ Female } \\
\hline 0 & $88.09 \pm 7.45$ & $85.85 \pm 5.38$ & $86.47 \pm 5.60$ & $87.40 \pm 6.06$ & $85.97 \pm 6.66$ & $88.00 \pm 6.54$ \\
\hline 12 & $260.35 \pm 17.67$ & $251.54 \pm 11.91$ & $247.59 \pm 21.34$ & $246.85 \pm 12.00$ & $257.96 \pm 10.36$ & $242.37 \pm 14.00$ \\
\hline 13 & $266.65 \pm 17.06$ & $257.29 \pm 12.04$ & $252.69 \pm 21.34$ & $252.47 \pm 11.99^{*}$ & $263.88 \pm 10.21$ & $246.83 \pm 13.20$ \\
\hline 17 & & & & & $267.14 \pm 7.43$ & $254.22 \pm 10.83^{*}$ \\
\hline
\end{tabular}

Data was represented as Mean \pm Standard Deviation (SD). Data was analyzed by unpaired ' $\mathrm{t}$ ' test, ${ }^{*}=\mathrm{P}<0.05$ as compared torespective VC groups.

Table 2: Effect of SFSE-G on hematological parameters during 90 days repeated dose toxicity study (Male rats).

\begin{tabular}{|c|c|c|c|c|c|c|}
\hline \multirow[t]{2}{*}{ Parameters } & G1 & G2 & G3 & G4 & G1R & G4R \\
\hline & $\overline{\mathrm{VC}}$ & SFSE-G-250 & SFSE-G-500 & SFSE-G-1000 & VC-R & SFSE-G-1000-R \\
\hline $\mathrm{Hb}(\mathrm{g} \%)$ & $15.86 \pm 0.84$ & $15.01 \pm 0.91^{* * *}$ & $15.76 \pm 0.60$ & $16.09 \pm 0.66$ & $17.94 \pm 1.48$ & $16.02 \pm 0.64 * *$ \\
\hline $\mathrm{RBC}(\mathrm{x} 106 / \mu \mathrm{L})$ & $8.61 \pm 0.52$ & $7.97 \pm 0.50 * *$ & $8.67 \pm 0.43$ & $8.85 \pm 0.55$ & $10.11 \pm 0.70$ & $8.89 \pm 0.37 * *$ \\
\hline Reticulocytes (\%) & $1.90 \pm 0.44$ & $1.65 \pm 0.39$ & $1.69 \pm 0.44$ & $1.73 \pm 0.38$ & $1.52 \pm 0.58$ & $1.50 \pm 0.43$ \\
\hline HCT (\%) & $43.15 \pm 2.07$ & $40.60 \pm 2.79 *$ & $43.59 \pm 1.83$ & $44.91 \pm 2.28$ & $49.12 \pm 3.36$ & $43.00 \pm 1.35^{* *}$ \\
\hline $\operatorname{MCV}\left(\mathrm{mm}^{3}\right)$ & $50.17 \pm 1.51$ & $50.95 \pm 1.15$ & $50.29 \pm 1.39$ & $50.79 \pm 1.34$ & $48.56 \pm 1.45$ & $48.44 \pm 1.85$ \\
\hline $\mathrm{MCH}(\mathrm{pg})$ & $18.47 \pm 0.69$ & $18.81 \pm 0.39$ & $18.19 \pm 0.58$ & $18.22 \pm 0.58$ & $17.72 \pm 0.33$ & $18.02 \pm 0.91$ \\
\hline $\mathrm{MCHC}(\%)$ & $36.79 \pm 0.49$ & $36.95 \pm 0.55$ & $36.19 \pm 0.46^{*}$ & $35.87 \pm 0.69 * *$ & $36.52 \pm 1.08$ & $37.26 \pm 0.58$ \\
\hline Platelets $\left(\times 10^{3} / \mu \mathrm{L}\right)$ & $416.20 \pm 66.93$ & $389.60 \pm 49.72$ & $436.47 \pm 72.72$ & $425.73 \pm 50.94$ & $391.20 \pm 69.19$ & $473.80 \pm 37.59 *$ \\
\hline $\mathrm{PT}(\mathrm{sec})$ & $15.53 \pm 3.23$ & $14.93 \pm 4.03$ & $13.93 \pm 3.33$ & $15.13 \pm 3.25$ & $16.20 \pm 3.42$ & $15.20 \pm 3.03$ \\
\hline $\operatorname{TLC}\left(\times 10^{3} / \mu \mathrm{L}\right)$ & $11.73 \pm 3.36$ & $10.49 \pm 2.11$ & $10.73 \pm 3.19$ & $10.61 \pm 2.14$ & $10.58 \pm 3.03$ & $10.58 \pm 1.16$ \\
\hline \multicolumn{7}{|c|}{ Differential Leucocyte Count } \\
\hline $\mathrm{N}(\%)$ & $21.33 \pm 2.69$ & $21.20 \pm 3.95$ & $21.13 \pm 4.14$ & $22.53 \pm 4.58$ & $20.80 \pm 3.27$ & $20.80 \pm 4.15$ \\
\hline $\mathrm{L}(\%)$ & $74.93 \pm 2.79$ & $75.60 \pm 3.87$ & $75.27 \pm 3.92$ & $73.87 \pm 4.52$ & $75.60 \pm 3.21$ & $76.00 \pm 3.81$ \\
\hline $\mathrm{E}(\%)$ & $1.13 \pm 0.74$ & $0.87 \pm 0.74$ & $1.27 \pm 0.70$ & $1.07 \pm 0.80$ & $1.20 \pm 0.84$ & $1.20 \pm 0.84$ \\
\hline $\mathrm{M}(\%)$ & $2.60 \pm 0.91$ & $2.33 \pm 0.72$ & $2.33 \pm 0.72$ & $2.53 \pm 0.83$ & $2.40 \pm 1.14$ & $2.00 \pm 0.71$ \\
\hline $\mathrm{B}(\%)$ & $0.00 \pm 0.00$ & $0.00 \pm 0.00$ & $0.00 \pm 0.00$ & $0.00 \pm 0.00$ & $0.00 \pm 0.00$ & $0.00 \pm 0.00$ \\
\hline
\end{tabular}

Data was represented as Mean \pm Standard Deviation, Data was analyzed by unpaired ' $\mathrm{t}$ ' test, $*=\mathrm{P}<0.05, * * \mathrm{P}<0.01$ as compared of respective parameter value of corresponding VC group. Hb: Hemoglobin; RBC: Red Blood Corpuscles, HCT : Hematocrit, MCV: Mean Corpuscular Volume, MCH: Mean Corpuscular Hemoglobin, MCHC: Mean Corpuscular Hemoglobin Concentration, TLC: Total leukocyte (White Blood Corpuscles) count, PT.: Prothrombin time, N: Neutrophils, L : Lymphocytes, E: Eosinophils, M : Monocytes, B : Basophils

Hematological (Table 2 and Table 3) and biochemical observations (Table 4 and Table 5) and urinalysis were recorded in male and female rats. All the values hematological and biochemical values were within normal biological and laboratory limits and the differences between the values were not consistent between doses or periods of observation (treatment period / reversal period) and considered incidental. For example, hematology values in SFSE-G treated groups of rats were significantly different from corresponding $\mathrm{VC}$ groups in terms of $\mathrm{Hb}, \mathrm{RBC}, \mathrm{HCT}, \mathrm{MCHC}$, TLC during 90 day treatment period and 
$\mathrm{Hb}, \mathrm{RBC}, \mathrm{HCT}$ and platelets during the recovery period (Table 2 and Table 3). Biochemical parameters related to kidney function test (CR, CK and BUN) did not show significant change as compared to respective $\mathrm{VC}$ group in female rats whereas BUN values show significant $(\mathrm{P}<0.05)$ decrease in $\mathrm{G} 2$ (SFSE-G-250 $\mathrm{mg} / \mathrm{kg}$ treated) group (Table 4).

Similarly, all liver function related biochemical parameters (ALT, AST, ALP, GGT, Bilirubin, Albumin) were unchanged in female rats whereas ALT values in G2 (SFSE-G-250 treated group) showed significant $(\mathrm{P}<0.05)$ increase in male rats (Table 4 and Table 5). The electrolyte concentrations ( $\mathrm{Ca}, \mathrm{P}, \mathrm{Na}, \mathrm{K}$ and $\mathrm{Cl}$ ) levels in male rats did not show significant changes as compared to respective VC groups except $\mathrm{Ca}$ levels (SFSE-G-1000 group, $\mathrm{P}<0.01$ ) and $\mathrm{Cl}$ levels (SFSE-G-250, $\mathrm{P}<0.01$ ). However, female rats showed significant changes in electrolyte concentration related parameters such as $\mathrm{Na}$ levels (SFSE-G-250 and SFSE-G-500 but not SFSE-G-1000 group), Ca and K levels (SFSE-G-500 and SFSE-G-1000 group), $\mathrm{Cl}$ levels (all SFSE-G treated groups) with no significant change in $P$ levels. The plasma lipid-related parameters (FPG, cholesterol, triglyceride, and LDH levels) in SFSE-G treated male and female rats remained unchanged except G2 (SFSE-G-250 treated) group which showed significant $(\mathrm{P}<0.05)$ decrease in triglyceride levels in female rats.

Table 3: Effect of SFSE-G on hematological parameters during 90 days repeated dose toxicity study (Female rats).

\begin{tabular}{|c|c|c|c|c|c|c|}
\hline \multirow{2}{*}{ Parameters } & G1 & G2 & G3 & G4 & G1R & G4R \\
\hline & VC & SFSE-G-250 & SFSE-G-500 & SFSE-G-1000 & VC-R & SFSE-G-1000-R \\
\hline $\mathrm{Hb}(\mathrm{g} \%)$ & $16.06 \pm 1.67$ & $15.41 \pm 0.65$ & $15.93 \pm 2.24$ & $15.10 \pm 0.82$ & $16.10 \pm 0.31$ & $15.66 \pm 0.98$ \\
\hline $\mathrm{RBC}(\mathrm{x} 106 / \mu \mathrm{L})$ & $8.27 \pm 0.87$ & $7.75 \pm 0.46$ & $8.16 \pm 1.16$ & $7.69 \pm 0.43^{*}$ & $8.39 \pm 0.31$ & $8.10 \pm 0.46$ \\
\hline Reticulocytes ( \%) & $1.83 \pm 0.35$ & $1.67 \pm 0.44$ & $1.63 \pm 0.41$ & $1.62 \pm 0.48$ & $1.48 \pm 0.48$ & $1.80 \pm 0.68$ \\
\hline $\operatorname{HCT}(\%)$ & $43.68 \pm 4.64$ & $41.25 \pm 1.76$ & $43.09 \pm 6.34$ & $40.77 \pm 2.21 *$ & $43.48 \pm 0.96$ & $41.82 \pm 2.58$ \\
\hline $\operatorname{MCV}\left(\mathrm{mm}^{3}\right)$ & $52.84 \pm 1.92$ & $53.29 \pm 2.28$ & $52.81 \pm 1.32$ & $53.01 \pm 1.35$ & $51.80 \pm 1.04$ & $51.62 \pm 0.99$ \\
\hline $\mathrm{MCH}(\mathrm{pg})$ & $19.43 \pm 0.83$ & $19.90 \pm 0.88$ & $19.56 \pm 0.57$ & $19.63 \pm 0.56$ & $19.22 \pm 0.83$ & $19.36 \pm 0.62$ \\
\hline $\mathrm{MCHC}(\%)$ & $36.80 \pm 0.89$ & $37.34 \pm 0.38 *$ & $37.04 \pm 1.24$ & $37.03 \pm 0.40$ & $37.10 \pm 1.02$ & $37.48 \pm 0.50$ \\
\hline Platelets $\left(\times 10^{3} / \mu \mathrm{L}\right)$ & $410.80 \pm 111.28$ & $441.47 \pm 59.52$ & $425.00 \pm 111.57$ & $409.80 \pm 80.62$ & $392.60 \pm 79.32$ & $440.00 \pm 86.80$ \\
\hline $\mathrm{PT}(\mathrm{sec})$ & $15.00 \pm 3.85$ & $13.93 \pm 3.84$ & $13.87 \pm 3.87$ & $15.20 \pm 3.47$ & $15.60 \pm 3.91$ & $16.40 \pm 3.58$ \\
\hline $\operatorname{TLC}\left(\times 10^{3} / \mu \mathrm{L}\right)$ & $12.44 \pm 4.06$ & $12.31 \pm 3.86$ & $9.63 \pm 3.20 *$ & $10.76 \pm 1.91$ & $9.46 \pm 2.88$ & $8.62 \pm 2.83$ \\
\hline \multicolumn{7}{|c|}{ Differential Leukocyte count } \\
\hline $\mathrm{N}(\%)$ & $20.93 \pm 4.06$ & $21.67 \pm 3.68$ & $21.47 \pm 4.12$ & $20.80 \pm 3.88$ & $21.00 \pm 3.39$ & $20.00 \pm 5.15$ \\
\hline $\mathrm{L}(\%)$ & $75.47 \pm 3.60$ & $74.80 \pm 3.00$ & $75.27 \pm 3.49$ & $75.73 \pm 3.39$ & $75.40 \pm 2.51$ & $76.40 \pm 4.10$ \\
\hline $\mathrm{E}(\%)$ & $1.13 \pm 0.83$ & $1.00 \pm 0.85$ & $1.00 \pm 0.76$ & $1.13 \pm 0.74$ & $1.20 \pm 0.84$ & $1.20 \pm 0.84$ \\
\hline $\mathrm{M}(\%)$ & $2.47 \pm 0.64$ & $2.53 \pm 0.64$ & $2.27 \pm 0.80$ & $2.33 \pm 0.72$ & $2.40 \pm 0.55$ & $2.40 \pm 0.55$ \\
\hline $\mathrm{B}(\%)$ & $0.00 \pm 0.00$ & $0.00 \pm 0.00$ & $0.00 \pm 0.00$ & $0.00 \pm 0.00$ & $0.00 \pm 0.00$ & $0.00 \pm 0.00$ \\
\hline
\end{tabular}

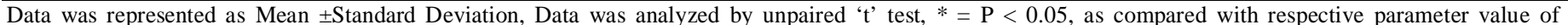

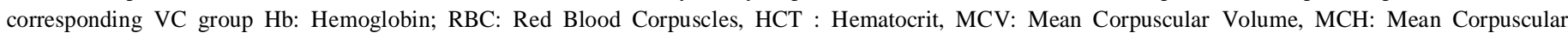

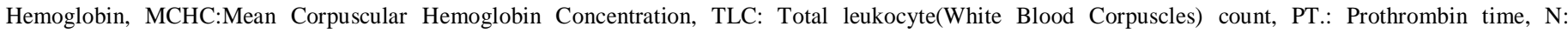
Neutrophils, L : Lymphocytes, E: Eosinophils, M : Monocytes, B : Basophils.

Table 4: Effect of SFSE-G on blood chemistry on during 90 days repeated dose toxicity study (Male rats).

\begin{tabular}{|c|c|c|c|c|c|c|}
\hline Parameters & $\begin{array}{l}\text { G1 } \\
\text { VC }\end{array}$ & $\begin{array}{l}\text { G2 } \\
\text { SFSE-G-250 }\end{array}$ & $\begin{array}{l}\text { G3 } \\
\text { SFSE-G-500 }\end{array}$ & $\begin{array}{l}\text { G4 } \\
\text { SFSE-G-1000 }\end{array}$ & $\begin{array}{l}\text { G1R } \\
\text { VC-R }\end{array}$ & $\begin{array}{l}\text { G4R } \\
\text { SFSE-G-1000-R }\end{array}$ \\
\hline \multicolumn{7}{|l|}{ Liver function Tests } \\
\hline ALT(IU/L) & $38.33 \pm 6.80$ & $43.47 \pm 4.00^{*}$ & $39.93 \pm 5.61$ & $40.20 \pm 5.76$ & $37.20 \pm 6.22$ & $33.40 \pm 2.19$ \\
\hline AST (IU/L) & $63.40 \pm 3.48$ & $64.47 \pm 4.24$ & $63.53 \pm 3.29$ & $63.33 \pm 3.81$ & $60.60 \pm 3.78$ & $63.60 \pm 3.51$ \\
\hline ALP (IU/L) & $72.93 \pm 4.10$ & $73.67 \pm 6.52$ & $71.33 \pm 6.28$ & $73.93 \pm 5.19$ & $70.00 \pm 4.64$ & $71.40 \pm 5.22$ \\
\hline GGT (U/L) & $14.73 \pm 4.45$ & $15.40 \pm 3.48$ & $18.00 \pm 3.23$ & $17.13 \pm 3.87$ & $15.20 \pm 3.56$ & $13.20 \pm 3.35$ \\
\hline Bilirubin (mg\%) & $0.67 \pm 0.07$ & $0.67 \pm 0.07$ & $0.65 \pm 0.09$ & $0.65 \pm 0.07$ & $0.69 \pm 0.08$ & $0.67 \pm 0.06$ \\
\hline Total Protein (g\%) & $7.68 \pm 0.64$ & $8.07 \pm 0.32$ & $7.88 \pm 0.41$ & $7.85 \pm 0.51$ & $7.50 \pm 0.60$ & $7.53 \pm 0.77$ \\
\hline Albumin $(\mathrm{g} \%)$ & $3.62 \pm 0.29$ & $3.79 \pm 0.24$ & $3.57 \pm 0.19$ & $3.56 \pm 0.26$ & $3.72 \pm 0.36$ & $3.59 \pm 0.32$ \\
\hline \multicolumn{7}{|l|}{ Kidney Function test } \\
\hline $\mathrm{CR}(\mathrm{mg} \%)$ & $0.96 \pm 0.11$ & $0.90 \pm 0.08$ & $0.95 \pm 0.09$ & $0.94 \pm 0.11$ & $0.95 \pm 0.06$ & $1.00 \pm 0.12$ \\
\hline CK (IU/L) & $62.00 \pm 4.19$ & $61.20 \pm 4.93$ & $61.27 \pm 3.75$ & $65.13 \pm 4.70$ & $64.80 \pm 5.02$ & $64.20 \pm 1.64$ \\
\hline BUN (mg\%) & $36.20 \pm 5.54$ & $32.00 \pm 2.88 *$ & $35.20 \pm 5.13$ & $34.87 \pm 4.37$ & $33.20 \pm 3.03$ & $29.60 \pm 5.55$ \\
\hline \multicolumn{7}{|l|}{ Serum Electrolytes } \\
\hline $\mathrm{Ca}(\mathrm{mg} \%)$ & $2.11 \pm 0.10$ & $2.16 \pm 0.10$ & $2.13 \pm 0.19$ & $2.27 \pm 0.08 * *$ & $2.36 \pm 0.11$ & $2.29 \pm 0.05$ \\
\hline $\mathrm{P}(\mathrm{mg} \%)$ & $4.25 \pm 0.39$ & $4.07 \pm 0.45$ & $4.40 \pm 0.45$ & $4.02 \pm 0.45$ & $4.04 \pm 0.32$ & $4.28 \pm 0.22$ \\
\hline $\mathrm{Na}(\mathrm{mmol} / \mathrm{l})$ & $139.56 \pm 1.81$ & $139.81 \pm 0.84$ & $139.79 \pm 0.95$ & $140.42 \pm 1.18$ & $138.00 \pm 0.97$ & $140.19 \pm 0.30 * *$ \\
\hline $\mathrm{K}(\mathrm{mmol} / \mathrm{l})$ & $4.55 \pm 0.24$ & $4.43 \pm 0.19$ & $4.42 \pm 0.30$ & $4.61 \pm 0.27$ & $4.30 \pm 0.21$ & $4.29 \pm 0.16$ \\
\hline $\mathrm{Cl}(\mathrm{mmol} / \mathrm{l})$ & $103.95 \pm 4.39$ & $99.24 \pm 5.15^{* *}$ & $107.37 \pm 3.36$ & $102.24 \pm 3.35$ & $107.20 \pm 1.61$ & $106.18 \pm 2.73$ \\
\hline \multicolumn{7}{|c|}{ Metabolic function tests } \\
\hline FPG (mg\%) & $98.27 \pm 5.78$ & $95.40 \pm 9.41$ & $93.67 \pm 9.04$ & $97.80 \pm 9.96$ & $88.20 \pm 6.14$ & $84.40 \pm 10.69$ \\
\hline Cholesterol (mg\%) & $61.07 \pm 6.37$ & $63.87 \pm 6.45$ & $63.40 \pm 6.03$ & $63.27 \pm 3.53$ & $64.60 \pm 3.51$ & $64.80 \pm 3.56$ \\
\hline Triglyceride $(\mathrm{mg} \%)$ & $107.53 \pm 5.63$ & $107.47 \pm 9.36$ & $110.20 \pm 9.16$ & $106.87 \pm 6.82$ & $111.40 \pm 10.01$ & $111.40 \pm 6.99$ \\
\hline LDH (IU/L) & $367.53 \pm 26.79$ & $363.07 \pm 37.67$ & $368.87 \pm 27.63$ & $359.60 \pm 23.46$ & $362.80 \pm 36.35$ & $340.40 \pm 22.68$ \\
\hline
\end{tabular}

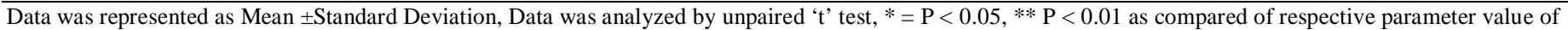

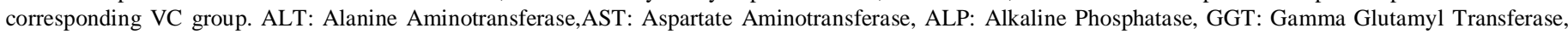

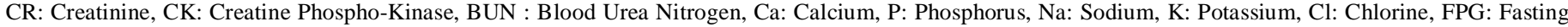
plasma glucose, LDH: Lactate De-Hydrogenase. 
Table 5: Effect of SFSE-G on blood chemistry on during 90 days repeated dose toxicity study (Female rats).

\begin{tabular}{|c|c|c|c|c|c|c|}
\hline \multirow{2}{*}{ Parameters } & G1 & G2 & G3 & G4 & G1R & G4R \\
\hline & VC & SFSE-G-250 & SFSE-G-500 & SFSE-G-1000 & VC-R & SFSE-G-1000-R \\
\hline \multicolumn{7}{|l|}{ Liver function Tests } \\
\hline ALT(IU/L) & $40.93 \pm 4.88$ & $41.00 \pm 4.74$ & $37.93 \pm 5.97$ & $40.53 \pm 5.93$ & $37.60 \pm 6.91$ & $41.60 \pm 5.13$ \\
\hline AST (IU/L) & $62.60 \pm 3.16$ & $63.80 \pm 4.28$ & $61.27 \pm 4.95$ & $62.93 \pm 4.59$ & $58.20 \pm 6.18$ & $63.40 \pm 3.36^{*}$ \\
\hline ALP (IU/L) & $73.27 \pm 5.08$ & $73.27 \pm 8.04$ & $71.47 \pm 7.85$ & $70.53 \pm 6.27$ & $70.00 \pm 5.20$ & $72.40 \pm 4.67$ \\
\hline GGT (U/L) & $18.47 \pm 3.02$ & $17.67 \pm 4.47$ & $16.93 \pm 3.45$ & $15.93 \pm 3.97$ & $17.20 \pm 5.26$ & $13.40 \pm 4.67$ \\
\hline Bilirubin (mg\%) & $0.67 \pm 0.07$ & $0.66 \pm 0.08$ & $0.67 \pm 0.08$ & $0.66 \pm 0.08$ & $0.67 \pm 0.07$ & $0.63 \pm 0.05$ \\
\hline Total Protein (g\%) & $7.56 \pm 0.48$ & $7.94 \pm 0.38$ & $7.68 \pm 0.58$ & $7.49 \pm 0.66$ & $7.33 \pm 0.56$ & $7.78 \pm 0.59$ \\
\hline Albumin $(\mathrm{g} \%)$ & $3.49 \pm 0.35$ & $3.59 \pm 0.34$ & $3.60 \pm 0.23$ & $3.53 \pm 0.21$ & $3.66 \pm 0.20$ & $3.57 \pm 0.35$ \\
\hline \multicolumn{7}{|c|}{ Kidney Function test } \\
\hline$\overline{\mathrm{CR}}(\mathrm{mg} \%)$ & $1.00 \pm 0.08$ & $1.01 \pm 0.10$ & $0.96 \pm 0.08$ & $0.98 \pm 0.08$ & $0.95 \pm 0.07$ & $0.96 \pm 0.05$ \\
\hline CK (IU/L) & $62.80 \pm 4.66$ & $64.00 \pm 3.87$ & $62.27 \pm 4.32$ & $61.87 \pm 4.45$ & $61.80 \pm 4.60$ & $61.80 \pm 4.49$ \\
\hline BUN (mg\%) & $35.27 \pm 5.65$ & $32.93 \pm 4.08$ & $34.07 \pm 4.15$ & $36.53 \pm 6.17$ & $33.60 \pm 1.82$ & $34.60 \pm 5.13$ \\
\hline \multicolumn{7}{|l|}{ Serum Electrolytes } \\
\hline $\mathrm{Ca}(\mathrm{mg} \%)$ & $2.45 \pm 0.14$ & $2.55 \pm 0.11$ & $2.26 \pm 0.14 *$ & $2.27 \pm 0.13^{*}$ & $2.29 \pm 0.12$ & $2.42 \pm 0.05^{*}$ \\
\hline $\mathrm{P}(\mathrm{mg} \%)$ & $4.12 \pm 0.41$ & $4.33 \pm 0.41$ & $4.13 \pm 0.45$ & $4.27 \pm 0.44$ & $3.98 \pm 0.38$ & $4.12 \pm 0.22$ \\
\hline $\mathrm{Na}(\mathrm{mmol} / \mathrm{l})$ & $138.13 \pm 1.81$ & $136.32 \pm 1.70 * *$ & $136.06 \pm 1.41 * *$ & $137.68 \pm 1.28$ & $136.74 \pm 0.84$ & $137.90 \pm 0.87 *$ \\
\hline $\mathrm{K}(\mathrm{mmol} / \mathrm{l})$ & $5.20 \pm 0.21$ & $5.22 \pm 0.39$ & $4.51 \pm 0.23 * *$ & $4.70 \pm 0.27 * *$ & $4.17 \pm 0.36$ & $4.09 \pm 0.34$ \\
\hline $\mathrm{Cl}(\mathrm{mmol} / \mathrm{l})$ & $96.97 \pm 15.59$ & $107.47 \pm 8.95^{*}$ & $128.12 \pm 9.18 * *$ & $131.31 \pm 15.78^{* * *}$ & $110.54 \pm 1.72$ & $111.53 \pm 2.03$ \\
\hline \multicolumn{7}{|c|}{ Metabolic function tests } \\
\hline FPG (mg\%) & $95.53 \pm 7.45$ & $96.80 \pm 8.77$ & $98.53 \pm 13.18$ & $98.87 \pm 10.97$ & $90.00 \pm 9.59$ & $96.80 \pm 13.16$ \\
\hline Cholesterol (mg\%) & $64.47 \pm 4.44$ & $65.27 \pm 3.97$ & $63.53 \pm 5.07$ & $64.53 \pm 4.12$ & $66.60 \pm 2.88$ & $64.60 \pm 4.39$ \\
\hline Triglyceride(mg\%) & $110.87 \pm 6.36$ & $105.73 \pm 4.92 *$ & $112.33 \pm 5.18$ & $110.13 \pm 10.20$ & $111.80 \pm 8.41$ & $114.20 \pm 6.22$ \\
\hline $\mathrm{LDH}(\mathrm{IU} / \mathrm{L})$ & $372.07 \pm 26.35$ & $357.07 \pm 27.16$ & $363.60 \pm 22.76$ & $375.07 \pm 13.32$ & $367.60 \pm 25.66$ & $383.00 \pm 19.52$ \\
\hline
\end{tabular}

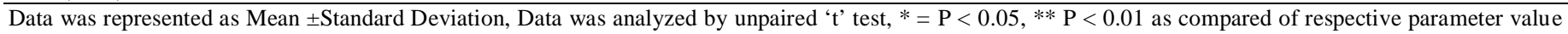

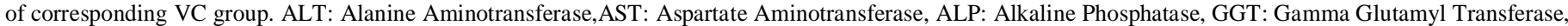

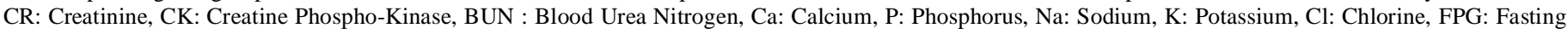
plasma glucose, LDH: Lactate De-Hydrogenase.

Table 6: Effect of SFSE-G on relative organ weights of during 90 days repeated dose toxicity study (Male rats).

\begin{tabular}{|c|c|c|c|c|c|c|}
\hline \multirow{2}{*}{ Organ } & G1 & G2 & G3 & G4 & G1R & G4R \\
\hline & $\overline{\mathrm{VC}}$ & SFSE-G-250 & SFSE-G-500 & SFSE-G-1000 & VC-R & SFSE-G-1000-R \\
\hline Brain & $0.561 \pm 0.037$ & $0.561 \pm 0.068$ & $0.561 \pm 0.042$ & $0.590 \pm 0.045$ & $0.529 \pm 0.027$ & $0.560 \pm 0.039$ \\
\hline Liver & $2.853 \pm 0.255$ & $2.835 \pm 0.279$ & $2.982 \pm 0.233$ & $2.957 \pm 0.164$ & $2.996 \pm 0.197$ & $3.033 \pm 0.278$ \\
\hline Kidneys & $0.725 \pm 0.053$ & $0.717 \pm 0.081$ & $0.724 \pm 0.056$ & $0.730 \pm 0.058$ & $0.731 \pm 0.049$ & $0.763 \pm 0.044$ \\
\hline Adrenals & $0.014 \pm 0.0013$ & $0.0131 \pm 0.0031$ & $0.0132 \pm 0.0018$ & $0.0140 \pm 0.0016$ & $0.013 \pm 0.002$ & $0.013 \pm 0.002$ \\
\hline Testes & $0.858 \pm 0.087$ & $0.794 \pm 0.104$ & $0.826 \pm 0.123$ & $0.896 \pm 0.087$ & $0.846 \pm 0.169$ & $0.823 \pm 0.057$ \\
\hline Heart & $0.335 \pm 0.031$ & $0.334 \pm 0.027$ & $0.333 \pm 0.027$ & $0.338 \pm 0.022$ & $0.322 \pm 0.014$ & $0.325 \pm 0.014$ \\
\hline Spleen & $0.336 \pm 0.055$ & $0.368 \pm 0.034$ & $0.358 \pm 0.065$ & $0.364 \pm 0.041$ & $0.323 \pm 0.031$ & $0.357 \pm 0.083$ \\
\hline Lungs & $0.466 \pm 0.055$ & $0.507 \pm 0.077$ & $0.486 \pm 0.068$ & $0.477 \pm 0.059$ & $0.455 \pm 0.057$ & $0.418 \pm 0.034$ \\
\hline Thymus & $0.049 \pm 0.010$ & $0.055 \pm 0.015$ & $0.055 \pm 0.014$ & $0.062 \pm 0.023$ & $0.031 \pm 0.007$ & $0.044 \pm 0.017$ \\
\hline Epididymis & $0.336 \pm 0.037$ & $0.325 \pm 0.033$ & $0.327 \pm 0.052$ & $0.360 \pm 0.036$ & $0.365 \pm 0.039$ & $0.365 \pm 0.023$ \\
\hline
\end{tabular}

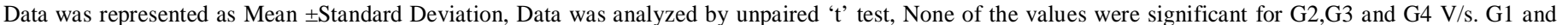
G4R v/s. G1R.

Table 7: Effect of SFSE-G on relative organ weights of during 90 days repeated dose toxicity study (Female rats)

\begin{tabular}{|c|c|c|c|c|c|c|}
\hline \multirow[t]{2}{*}{ Organ } & G1 & G2 & G3 & G4 & G1R & G4R \\
\hline & $\mathrm{VC}$ & SFSE-G-250 & SFSE-G-500 & SFSE-G-1000 & VC-R & SFSE-G-1000-R \\
\hline Brain & $0.749 \pm 0.040$ & $0.780 \pm 0.047$ & $0.785 \pm 0.052$ & $0.777 \pm 0.031$ & $0.764 \pm 0.032$ & $0.785 \pm 0.028$ \\
\hline Liver & $3.156 \pm 0.270$ & $3.235 \pm 0.343$ & $3.276 \pm 0.190$ & $3.372 \pm 0.345$ & $3.155 \pm 0.246$ & $3.255 \pm 0.335$ \\
\hline Kidneys & $0.652 \pm 0.043$ & $0.634 \pm 0.090$ & $0.690 \pm 0.059$ & $0.678 \pm 0.040$ & $0.685 \pm 0.059$ & $0.695 \pm 0.049$ \\
\hline Adrenals & $0.0210 \pm 0.0025$ & $0.0228 \pm 0.0039$ & $0.0225 \pm 0.0023$ & $0.0221 \pm 0.0025$ & $0.022 \pm 0.003$ & $0.021 \pm 0.002$ \\
\hline Ovaries & $0.0382 \pm 0.0054$ & $0.0358 \pm 0.0052$ & $0.0412 \pm 0.0035$ & $0.0384 \pm 0.0056$ & $0.0322 \pm 0.0052$ & $0.0357 \pm 0.0037$ \\
\hline Heart & $0.336 \pm 0.017$ & $0.346 \pm 0.032$ & $0.347 \pm 0.027$ & $0.346 \pm 0.023$ & $0.342 \pm 0.039$ & $0.354 \pm 0.023$ \\
\hline Spleen & $0.423 \pm 0.073$ & $0.419 \pm 0.076$ & $0.437 \pm 0.083$ & $0.433 \pm 0.068$ & $0.354 \pm 0.065$ & $0.350 \pm 0.054$ \\
\hline Lungs & $0.566 \pm 0.084$ & $0.604 \pm 0.054$ & $0.578 \pm 0.044$ & $0.592 \pm 0.052$ & $0.582 \pm 0.045$ & $0.609 \pm 0.053$ \\
\hline Thymus & $0.084 \pm 0.023$ & $0.098 \pm 0.016$ & $0.101 \pm 0.025$ & $0.098 \pm 0.022$ & $0.065 \pm 0.009$ & $0.068 \pm 0.014$ \\
\hline Uterus & $0.141 \pm 0.033$ & $0.163 \pm 0.041$ & $0.134 \pm 0.037$ & $0.160 \pm 0.031$ & $0.151 \pm 0.044$ & $0.160 \pm 0.035$ \\
\hline
\end{tabular}

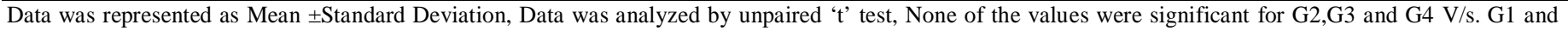
G4R v/s. G1R.

The data of weights of organs in SFSE-G treated rats during treatment and reversal period in male and female rats is presented in Table 6 and Table 7. None of the SFSE-G treated group show significant difference during treatment or reversal period as compared with corresponding VC group.No treatment-related gross pathological changes were observed in any organs of the test animals during necropsy (Table 8). 
Table 8: Summary of gross pathology findings.

\begin{tabular}{|c|c|c|c|c|c|c|c|c|c|c|c|c|}
\hline \multirow[t]{2}{*}{ Parameter } & \multicolumn{2}{|c|}{ G1 } & \multicolumn{2}{|c|}{ G2 } & \multicolumn{2}{|c|}{ G3 } & \multicolumn{2}{|c|}{ G4 } & \multicolumn{2}{|c|}{ G1R } & \multicolumn{2}{|c|}{ G4R } \\
\hline & & & SFS & -250 & SFS & -500 & SFSE & 1000 & & & SFSE- & 000-R \\
\hline $\begin{array}{c}\text { Sex } \\
\text { Organs/lesions }\end{array}$ & $\begin{array}{c}\mathrm{M} \\
\mathrm{NAD}\end{array}$ & $\begin{array}{c}\mathrm{F} \\
\mathrm{NAD}\end{array}$ & $\begin{array}{c}\mathrm{M} \\
\mathrm{NAD}\end{array}$ & $\begin{array}{c}\mathrm{F} \\
\mathrm{NAD}\end{array}$ & $\begin{array}{c}\mathrm{M} \\
\mathrm{NAD}\end{array}$ & $\begin{array}{c}\mathrm{F} \\
\mathrm{NAD}\end{array}$ & $\begin{array}{c}\text { M } \\
\text { NAD }\end{array}$ & $\begin{array}{c}\mathrm{F} \\
\mathrm{NAD}\end{array}$ & $\begin{array}{c}M \\
\text { NAD }\end{array}$ & $\begin{array}{c}\mathrm{F} \\
\mathrm{NAD}\end{array}$ & $\begin{array}{c}\mathrm{M} \\
\mathrm{NAD}\end{array}$ & $\begin{array}{c}\mathrm{F} \\
\mathrm{NAD}\end{array}$ \\
\hline
\end{tabular}

$\mathrm{M}=$ Male, $\mathrm{F}=$ Female, NAD = No Abnormality Detected.

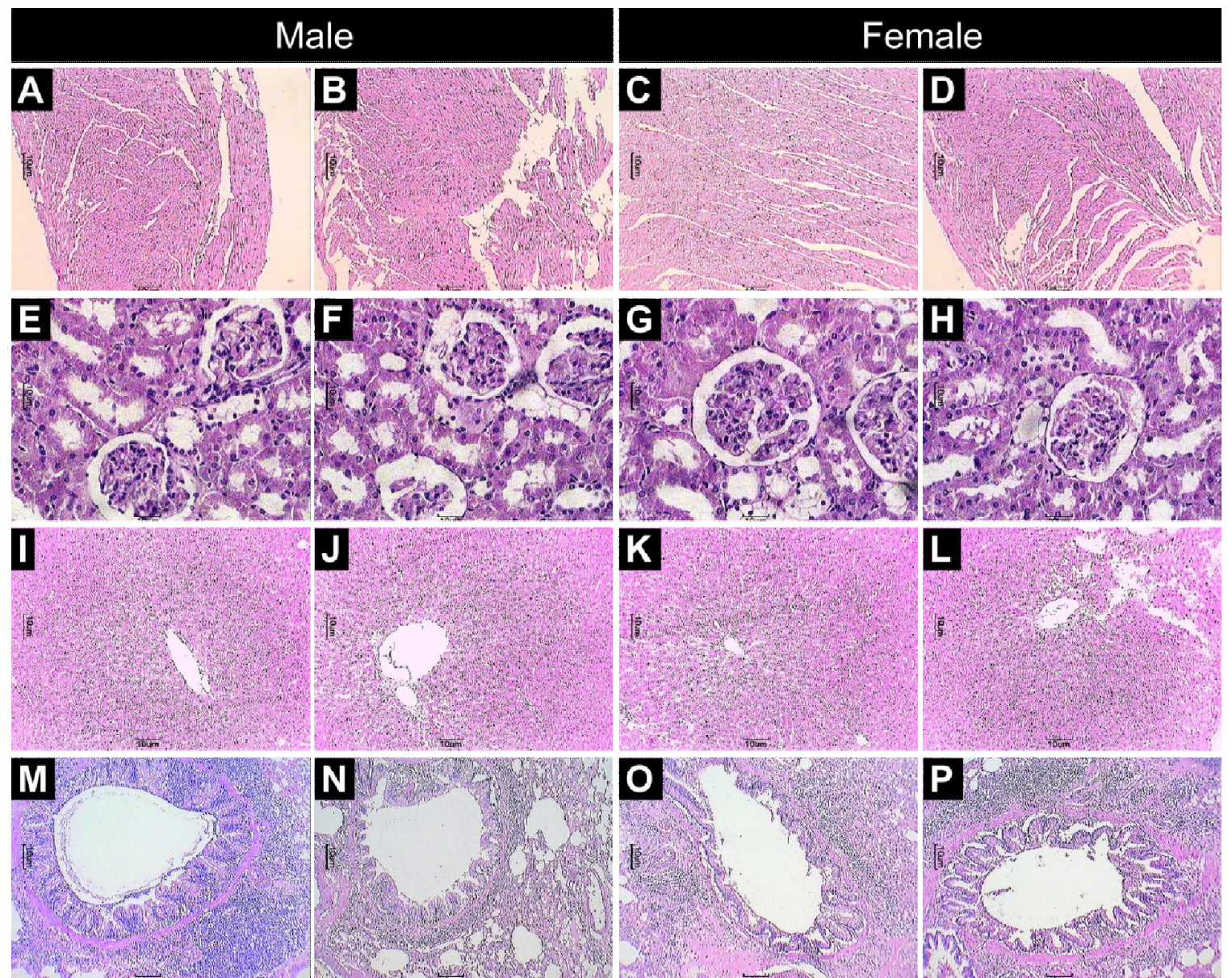

Fig. 2: Effects of SFSE-G on histological findings of heart (A-D), kidney (E-H), liver (I-L) and lung (M-P) tissue in rats during 90- days repeated dose toxicity study. Photomicrographs from representative rats from respective groups: VC (A, C, E, G, I, K, M, and O) and SFSE-G-1000 (B, D, F, H, J, L, N and P) (H\&E stain) at $40 \mathrm{X}$

The finding from histological examination of sections of organs is presented in Table 9 and representative photomicrographs are presented as Figure 2. Histopathological examination of the VC group and SFSE-G treated rats showed anormal structure with mild changes and absence of any gross pathological lesion in organs. The changes observed in both VC and SFSE-G1000 treatment groups were similar and comparable in both sexes and hence considered as incidental, congenital, and spontaneous. Based on the results of present subchronic toxicity study of SFSE$\mathrm{G}$, the no observed adverse effect level (NOAEL) is $1000 \mathrm{mg} / \mathrm{kg}$ per day in male rats and $500 \mathrm{mg} / \mathrm{kg}$ per day in female rats.

\section{Mutagenicity}

The numbers of reverse mutation colonies for the positive control substances such as sodium azide (NaN3), 2-acetamidofluorene (2-AF), 4-Nitroquinolene-N-Oxide (NQNO), Methyl methane sulphonate (MMS), 2 - Aminoanthracene (2-AA) and danthron showed significant increases in the revertant count than the negative control.

Thus, the test strains fulfilled the quality check criteria. No substantial increases in the revertant colony count in any of the five strains were reported in the SFSE-G treated plates in the presence or absence of metabolic activation (S9 mix).

The spontaneous reversion rates in the negative and positive controls were within the range of historical data. The results of these investigations suggest that under the experimental conditions, SFSE-G did not induce gene mutation by pair changes or frame-shifts in the genome of the strains used. Thus, SFSE-G can be considered as non-mutagenic. 
Table 9: Summary of histopathology findings.

\begin{tabular}{|c|c|c|c|c|}
\hline \multirow{3}{*}{$\begin{array}{l}\text { Sex } \\
\text { Parameters }\end{array}$} & \multicolumn{2}{|c|}{ Male } & \multicolumn{2}{|c|}{ Female } \\
\hline & & SFSE & & SFSE \\
\hline & VC & $\begin{array}{c}-G- \\
1000\end{array}$ & VC & $\begin{array}{c}-G- \\
1000\end{array}$ \\
\hline Number of Animals examined: & 15 & 15 & 15 & 15 \\
\hline Adrenals & NA & NA & NA & NA \\
\hline Dilation, sinusoidal & 3 & 2 & 3 & 2 \\
\hline Aorta & 0 & 0 & 0 & 0 \\
\hline Brain & NA & NA & NA & NA \\
\hline Lymphocytic Infiltration & 0 & 0 & 1 & 0 \\
\hline Necrosis & 2 & 0 & 1 & 1 \\
\hline Caecum & 0 & 0 & 0 & 0 \\
\hline Colon & 0 & 0 & 0 & 0 \\
\hline Duodenum & 0 & 0 & 0 & 0 \\
\hline Epididymis & 0 & 0 & NA & NA \\
\hline Eyes & 0 & 0 & 0 & 0 \\
\hline Heart & 0 & 0 & 0 & 0 \\
\hline Ileum & 0 & 0 & 0 & 0 \\
\hline Jejunum & 0 & 0 & 0 & 0 \\
\hline Kidneys & NA & NA & NA & NA \\
\hline Lymphocyte Infiltration, interstitial & 1 & 1 & 3 & 4 \\
\hline Necrosis & 0 & 1 & 2 & 0 \\
\hline Liver & NA & NA & NA & NA \\
\hline Lymphocyte Infiltration, periportal & 14 & 13 & 15 & 15 \\
\hline Necrosis & 1 & 0 & 0 & 1 \\
\hline Lungs & NA & NA & NA & NA \\
\hline Pneumonitis & 15 & 15 & 15 & 15 \\
\hline Haemorrhages & 4 & 6 & 7 & 3 \\
\hline Histiocytosis & 1 & 2 & 0 & 2 \\
\hline Lymph Node & NA & NA & NA & NA \\
\hline Cyst & 0 & 0 & 0 & 1 \\
\hline Ovaries & NA & NA & NA & NA \\
\hline Skin & 0 & 0 & 0 & 0 \\
\hline Oesophagus & 0 & 0 & 0 & 0 \\
\hline Pancreas & NA & NA & NA & NA \\
\hline LymphocyteInfiltration & 0 & 0 & 0 & 1 \\
\hline Pituitary & NA & NA & NA & NA \\
\hline Cysts & 3 & 0 & 2 & 1 \\
\hline Prostate & 0 & 0 & NA & NA \\
\hline Rectum & 0 & 0 & 0 & 0 \\
\hline Sciatic nerve & 0 & 0 & 0 & 0 \\
\hline Seminal vesicles & 0 & 0 & NA & NA \\
\hline Skeletal muscle & 0 & 0 & 0 & 0 \\
\hline Spleen & 0 & 0 & 0 & 0 \\
\hline Spinal cord & 0 & 0 & 0 & 0 \\
\hline Sternum with bone marrow & 0 & 0 & 0 & 0 \\
\hline Stomach & NA & NA & NA & NA \\
\hline Dilatation, glandular & 0 & 0 & 0 & 1 \\
\hline Testes & 0 & 0 & NA & NA \\
\hline Trachea & NA & NA & NA & NA \\
\hline Lymphocytic Infiltration, submucosal & 6 & 10 & 6 & 8 \\
\hline Thymus & 0 & 0 & 0 & 0 \\
\hline Thyroid / Parathyroid & NA & NA & NA & NA \\
\hline Ultimobranchial Cysts & 3 & 6 & 1 & 2 \\
\hline Urinary Bladder & 0 & 0 & 0 & 0 \\
\hline Uterus & NA & NA & NA & NA \\
\hline Eosinophilic infiltration & NA & NA & 14 & 15 \\
\hline
\end{tabular}

$\mathrm{NA}=$ Not applicable.

\section{DISCUSSION}

Toxicology studies are the platform for hazard identification stage of safety assessment (Wallace, 2011). The preclinical toxicity testing with safe dose level determination is needed to initiate the clinical evaluation of investigational products (Setzer and Kimmel, 2003). Choosing the appropriate tests and dosing regimens that will demonstrate an adequate margin of exposure is a critical step in establishing human safety. Toxicity data from studies following well-accepted international guidelines in animals is required by regulatory agencies throughout the world to support regulatory applications and marketing approval of natural products.

During the evaluation of the safety information of plants derived natural products, the determination of median lethal dose $\left(\mathrm{LD}_{50}\right)$ is usually an initial step to be conducted. Data from the acute toxicity study may provide initial information on the mode of toxic action of a substance, help in dose determination in animal studies (Ukwuani et al., 2012). In the present study, acute administration of SFSE-G at a dose of $2000 \mathrm{mg} / \mathrm{kg}$, showed no adverse effects up to 14 days and so considered as the median lethal dose $\left(\mathrm{LD}_{50}\right)$.

Based on results of AOT, subchronic (90-days repeated dose) toxicity study of SFSE-G was conducted in rats. Subchronic studies assess the undesirable effects of repeated long-term exposure of test product over a portion of the average life span of experimental animals, such as rodents. Therefore, they provide useful information on target organ toxicity and help to determine appropriate dose regimens for long-term use. Administration of SFSE-G for 90 days produced no clinical signs of toxicity or mortality in either sex.

The body weight changes serve as a sensitive indication of the general health status of animals (El Hilaly et al., 2004). Loss of appetite is often synonymous with weight loss due to disturbances in carbohydrate, protein or fat metabolisms (Klaassen, 2013). At higher doses, plant extracts may metabolize to a toxic end product, which could interfere with gastric function and decreased food conversion efficiency (Chokshi, 2007). In the present study, SFSE-G treated groups of rats showed body weight gain (although statistically not significant). Furthermore, the food consumption of SFSE-G treated rats remained unaltered during the 90-day treatment period. These results indicated the absence of adverse effects on the normal metabolism of food components during the treatment period and maintained expected nutritional benefits of normal food and water, such as weight gain and stability of the appetite.

Evaluation of hematological parameters can be used to determine the extent of the deleterious effect of SFSE-G on the blood of an animal(Chukwuemeka et al., 2015).Furthermore, such analysis is relevant to risk evaluation as changes in the hematological system have higher predictive value for human toxicity when the data are translated from animal studies (Chanda et al., 2015, Diaz et al., 2016).

A hematology data of SFSE-G treated rats did not show treatment-related changes as compared to $\mathrm{VC}$ rats. The results of hematology indicated the safety of SFSE-G on erythropoiesis, morphology or osmotic fragility of the red blood cells (Hall, 2015). Leukocytes are the first line of cellular defense that respond to infectious agents, tissue injury, or inflammatory process. The absence of significant treatment-related changes on TLC or differential leukocyte parameters in SFSE-G treated rats in the present study confirmed the safety of SFSE-G. 
A normal hematological profile of SFSE-G was further supported by the absence of adverse effects on biochemical parameters. Being vital to the survival of an organism, liver and kidney function tests are important in any toxicity evaluation program of drugs and plant extracts (Avigan et al., 2016, Stickel and Shouval, 2015). High levels of ALT, AST, and ALP indicate hepatotoxicity and possibility of liver diseases (Simpson and Freshwater, 2015). A decrease in total protein and albumin is a sign of the reduced protein synthesis, impaired hepatocellular function, infection or continuous loss of albumin (Cabrerizo et al., 2015, Waidely et al., 2016).

The absence of treatment-related adverse effects on liver function parameters in SFSE-G treated rats in the present study suggests safety of subchronic administration of SFSE-G treatment on liver or hepatocyte functions in the rats.

The weighing dissected organs in toxicity studies are useful to assess their sensitivity to test compound and a target organ of toxicity in terms of possible enzyme induction, physiologic perturbations, and acute injury (Michael et al., 2007). The results of present study revealed the absence of target organ toxicity by subchronic administration of SFSE-G. The absolute and relative weights of the essential organs (heart, liver, spleen, kidneys, and lungs) were neither adversely affected nor showed clinical signs of toxicity throughout the subchronic treatment of SFSE-G in the tested dose range. The safety of SFSE-G on essential organ toxicity correlates well with histological findings with little inter-animal variability. The photomicrographs from sections of vital organs (heart, liver, spleen, kidney, and lung) did not show any alterations in cell structure, unfavorable effects or pathological changes.

In the present study, recovery group, SFSE-G-1000-R (treated with limit dose, $1000 \mathrm{mg} / \mathrm{kg}$ ) did not show the occurrence of systemic toxic effects during recovery period (28 days after treatment period of 90 days) as compared to VC-R group. The absence of toxic effects or any reversibility in SFSE-1000-R group confirmed nontoxic nature of SFSE-G.

To evaluate mutagenicity potential of SFSE-G, the reverse mutation assay (AMES test) (Mortelmans and Zeiger, 2000)was used in the present study. The SFSE-G, over a broad concentration range $(61.72-5000 \mu \mathrm{g} /$ plate, did not show mutagenicity either presence or absence of a metabolic activator.

In the past, subchronic toxicity safety studies of glycosides from other plant sources were reported. For example, glycosides from Stevia rebaudiana were found safe during subchronic toxicity study in Wistar rats (Curry and Roberts, 2008). Furthermore, purified fraction of rhamnoside, a glycoside, from Pteranthus dichotomus found to be safe for acute administration in rats (Atta et al., 2013). The results of the present study are in line with reports of the safety of glycosides isolated from other sources. Human equivalent dose (HED) can be derived from NOAEL by using USFDA guidance for Industry (Center for Drug Evaluation and Research, 2005). Based on results of the present study (NOAEL of $500 \mathrm{mg} / \mathrm{kg}$ in rats, HED of SFSE-G is can be calculated to $4.8 \mathrm{~g} / \mathrm{day}$ (for the average human weight of $60 \mathrm{~kg}$ ).
The HED of $4.8 \mathrm{~g} /$ day is 8 times higher than efficacy dose of 600 $\mathrm{mg}$ /day during a clinical study in male healthy subjects (Mokashi et al., 2014) confirming broad margin of safety of SFSE-G.

\section{CONCLUSION}

The present study demonstrated the preclinical safety of SFSE-G during acute and subchronic (90-days repeated dose) administration in laboratory rats without mutagenicity potential in vitro. The median lethal dose cut-off value of SFSE-G was more than $2000 \mathrm{mg} / \mathrm{kg}$ and NOAEL of $1000 \mathrm{mg} / \mathrm{kg}$ and $500 \mathrm{mg} / \mathrm{kg}$ in male and female rats respectively.

\section{ACKNOWLEDGEMENT}

The authors acknowledge Indian Institute of Toxicology, Pune, India for their research services for the study.

\section{Financial support and sponsorship: Nil.}

Conflict of Interests: There are no conflicts of interest.

\section{REFERENCES}

Aswar U, Bodhankar S, Thakurdesai P, Mohan V. Androgenic and anabolic activity of glycoside based standardized fenugreek seeds extract on male Wistar rats. APTICON2014- 19th Annual National Convention of Association of Pharmaceutical Teachers of India. Pune, India: APTI, India; 2014.

Aswar U, Bodhankar SL, Mohan V, Thakurdesai PA. Effect of furostanol glycosides from Trigonella foenum-graecum on the reproductive system of male albino rats. Phytother Res. 2010; 24:1482-8.

Atta EM, Nassar AA, Hasan NM, Hasan AR. New flavonoid glycoside and pharmacological activities of Pteranthus dichotomus Forssk. Records of Natural Products. 2013; 7.

Avigan MI, Mozersky RP, Seeff LB. Scientific and Regulatory Perspectives in Herbal and Dietary Supplement Associated Hepatotoxicity in the United States. Int J Mol Sci. 2016; 17:331.

Basch E, Ulbricht C, Kuo G, Szapary P, Smith M. Therapeutic applications of fenugreek. Altern Med Rev. 2003; 8:20-7.

Cabrerizo S, Cuadras D, Gomez-Busto F, Artaza-Artabe I, Marin-Ciancas F, Malafarina V. Serum albumin and health in older people: Review and meta analysis. Maturitas. 2015; 81:17-27.

Center for Drug Evaluation and Research. Guidance for Industry: Estimating the maximum safe starting dose in initial clinical trials for therapeutics in adult healthy volunteers. US Department of Health and Human Services, Food and Drug Administration. 2005.

Chanda S, Parekh J, Vaghasiya Y, Dave R, Baravalia Y, Nair R. Medicinal plants-from traditional use to toxicity assessment: A Review. International Journal of Pharmaceutical Sciences and Research. 2015; $6: 2652$.

Chokshi D. Subchronic oral toxicity of a standardized white kidney bean (Phaseolus vulgaris) extract in rats. Food Chem Toxicol. 2007; 45:32-40.

Curry LL, Roberts A. Subchronic toxicity of rebaudioside A. Food Chem Toxicol. 2008; 46 Suppl 7:S11-20.

Diaz D, Hartley DP, Kemper R. Issue Investigation and Practices in Discovery Toxicology. Drug Discovery Toxicology: From Target Assessment to Translational Biomarkers. 2016:530-9.

El Hilaly J, Israili ZH, Lyoussi B. Acute and chronic toxicological studies of Ajuga iva in experimental animals. Journal of ethnopharmacology. 2004; 91:43-50.

Gupta RK, Jain DC, Thakur RS. Furostanol glycosides from Trigonella foenum-graecum seeds. Phytochemistry. 1985; 24:2399-401. 
Hall JE. Guyton and Hall textbook of medical physiology. 13th ed: Elsevier Health Sciences; 2015.

Han Y, Nishibe S, Noguchi Y, Jin Z. Flavonol glycosides from the stems of Trigonella foenum-graecum. Phytochemistry. 2001; 58:57780 .

Huang W, Liang X. Determination of two flavone glycosides in the seeds of Trigonella foenum-graecum $\mathrm{L}$. from various production locality. Journal of Plant Resources and Environment. 1999; 9:53-4.

Kalaiselvan V, Saurabh A, Kumar R, Singh GN. Adverse reactions to herbal products: An analysis of spontaneous reports in the database of the Pharmacovigilance Programme of India. Journal of herbal medicine. 2015; 5:48-54.

Kandhare AD, Bodhankar SL, Mohan V, Thakurdesai PA. Acute and repeated doses (28 days) oral toxicity study of glycosides based standardized fenugreek seed extract in laboratory mice. Regul Toxicol Pharmacol. 2015; 72:323-34.

Kang L-p, Zhao Y, Pang X, Yu H-s, Xiong C-q, Zhang J, et al Characterization and identification of steroidal saponins from the seeds of Trigonella foenum-graecum by ultra high-performance liquid chromatography and hybrid time-of-flight mass spectrometry. J Pharm Biomed Anal. 2013; 74:257-67.

Kawabata T, Cui MY, Hasegawa T, Takano F, Ohta T. Antiinflammatory and anti-melanogenic steroidal saponin glycosides from Fenugreek (Trigonella foenum-graecum L.) seeds. Planta Medica. 2011; 77:705-10.

Kenny O, Smyth TJ, Hewage CM, Brunton NP. Antioxidant properties and quantitative UPLC-MS analysis of phenolic compounds from extracts of fenugreek (Trigonella foenum-graecum) seeds and bitter melon (Momordica charantia) fruit. Food chemistry. 2013; 141:4295-302.

Klaassen CD. Casarett and Doull's toxicology: the basic science of poisons: McGraw-Hill New York (NY); 2013.

Michael B, Yano B, Sellers RS, Perry R, Morton D, Roome N, et al. Evaluation of organ weights for rodent and non-rodent toxicity studies: a review of regulatory guidelines and a survey of current practices. Toxicol Pathol. 2007; 35:742-50.

Mokashi M, Singh-Mokashi R, Mohan V, Thakurdesai PA. Effects of glycosides based fenugreek seed extract on serum testosterone levels of healthy sedentary male subjects: A exploratory double blind, placebo controlled, crossover study. Asian Journal of Pharmaceutical and Clinical Research. 2014; 7:177-81.

Mortelmans K, Zeiger E. The Ames Salmonella/microsome mutagenicity assay. Mutat Res. 2000; 455:29-60.

Murakami T, Kishi A, Matsuda H, Yoshikawa M. Medicinal foodstuffs. XVII. Fenugreek seed. (3): structures of new furostanol-type steroid saponins, trigoneosides Xa, Xb, XIb, XIIa, XIIb, and XIIIa, from the seeds of Egyptian Trigonellafoenum-graecum L. Chem Pharm Bull (Tokyo). 2000; 48:994-1000.

Organisation for Economic Co-operation and Development. Test No. 471: Bacterial Reverse Mutation Test. OECD Guidelines for the Testing of Chemicals, Section 4: Health Effects. Paris: OECD Publishing; 1997.

Organisation for Economic Co-operation and Development. Test No. 423: Acute Oral toxicity - Acute Toxic Class Method. OECD Guidelines for the Testing of Chemicals, Section 4: Health Effects. Paris: OECD Publishing; 2002.

Organisation for Economic Co-operation and Development. Test No. 408: Repeated Dose 90-Day Oral Toxicity Study in Rodents. OECD Guidelines for the Testing of Chemicals, Section 4: Health Effects. Paris: OECD Publishing; 1998. p. 1 online resource ( v.).

Pang X, Cong Y, Yu H-S, Kang L-P, Feng B, Han B-X, et al. Spirostanol saponins derivated from the seeds of Trigonella foenumgraecum by $\beta$-glucosidase hydrolysis and their inhibitory effects on rat platelet aggregation. Planta Med. 2012a; 78:276-85.

Pang X, Kang L-P, Yu H-S, Zhao Y, Xiong C-Q, Zhang J, et al. New kaurene diterpenoid glycosides from fenugreek seeds. Nat Prod Res. $2012 b$.

Petropoulos GA. Fenugreek: the genus Trigonella: CRC Press;
Rayyan S, Fossen T, Andersen ØM. Flavone C-glycosides from seeds of fenugreek, Trigonella foenum-graecum L. J Agric Food Chem. 2010; 58:7211-7.

Sauvaire Y, Ribes G, Baccou JC, Loubatieeres-Mariani MM. Implication of steroid saponins and sapogenins in the hypocholesterolemic effect of fenugreek. Lipids. 1991; 26:191-7.

Setzer RW, Kimmel CA. Use of NOAEL, benchmark dose, and other models for human risk assessment of hormonally active substances. Pure and applied chemistry. 2003; 75:2151-8.

Shang M, Cai S, Han J, Li J, Zhao Y, Zheng J, et al. [Studies on flavonoids from Fenugreek (Trigonella foenumgraecum L.)]. Zhongguo Zhong Yao Za Zhi. 1998; 23:614-6, 39.

Simpson MA, Freshwater DA. The interpretation and management of abnormal liver function tests. J R Nav Med Serv. 2015; 101:74-9.

Starr RR. Too little, too late: ineffective regulation of dietary supplements in the United States. Am J Public Health. 2015; 105:478-85.

Stickel F, Shouval D. Hepatotoxicity of herbal and dietary supplements: an update. Arch Toxicol. 2015; 89:851-65.

Taylor WG, Elder JL, Chang PR, Richards KW. Microdetermination of diosgenin from fenugreek (Trigonella foenumgraecum) seeds. J Agric Food Chem. 2000; 48:5206-10.

Ukwuani A, Abubakar M, Hassan S, Agaie B. Toxicological studies of hydromethanolic leaves extract of Grewia crenata. Int J Pharm Sci Drug Res. 2012; 4:245-9.

Ulbricht C, Basch E, Burke D, Cheung L, Ernst E, Giese N, et al. Fenugreek (Trigonella foenum-graecum L. Leguminosae): an evidencebased systematic review by the natural standard research collaboration. J Herbal Pharmacother. 2008; 7:143-77.

Upton R, Graff A, Jolliffe G, Länger R, Williamson E. American Herbal Pharmacopoeia: Botanical Pharmacognosy-Microscopic Characterization of Botanical Medicines: CRC Press; 2016.

Wagner H, Iyengar MA, Hörhammer L. Vicenin-1 and-2 in the seeds of Trigonella foenumgraecum. Phytochemistry. 1973; 12:2548.

Waidely E, Al-Yuobi AR, Bashammakh AS, El-Shahawi MS, Leblanc RM. Serum protein biomarkers relevant to hepatocellular carcinoma and their detection. Analyst. 2016; 141:36-44.

Wallace HM. Risk Perception in Toxicology-Part II: Toxicology Must Be the Solution Not the Problem. Toxicol Sci. 2011; 121:7-10.

Wilborn C, Taylor L, Poole C, Foster C, Willoughby D, Kreider R. Effects of a Purported Aromatase and $5 \alpha$-Reductase Inhibitor on Hormone Profiles in College-Age Men. Int J Sport Nutr. 2010; 20:457.

Yau WP, Goh CH, Koh HL. Quality Control and Quality assurance of Phytomedicines: Key Considerations, methods, and analytical challenges. Phytotherapies: Efficacy, Safety, and Regulation. 2015:18.

Yoshikawa M, Murakami T, Komatsu H, Murakami N, Yamahara J, Matsuda H. Medicinal foodstuffs. IV. Fenugreek seed. (1): structures of trigoneosides Ia, Ib, IIa, IIb, IIIa, and IIIb, new furostanol saponins from the seeds of Indian Trigonella foenum-graecum L. Chem Pharm Bull (Tokyo). 1997; 45:81-7.

\section{How to cite this article:}

Deshpande P, Mohan V, Thakurdesai P. Preclinical safety assessment of glycosides based standardized fenugreek seeds extract: Acute, subchronic toxicity and mutagenicity studies. J App Pharm Sci, 2016; 6 (09): 179-188. 